not continuously, but onitting it for a few days. At the full period labour commenced and progressed favourably, although her doubts and anxiety were greater than before. Immediately before the expulsion of the head I gave a dose of the ergot; a firm supporter was ready to be applied; the child was born; in fifteen ninutes the placenta was expelled; and there was no more discharge than in the most common and favourable cases, and she very quickly recovered.

My second case was similar to the above, except that her floodings had never been so severe. The oxide was given for four weeks in the latter part of November and the beginning of December. On the 25th ult. she was delivered of a healthy child. There was no hæmorrhage; and yesterday, the 2nd of January, she was so well as to express a desire to get out of bed.

The results in these two cases may, or may not be, in consequence of the administration of the oxide of silver; but $I$ would ask my medical brethren to make a trial of the remedy in patients where their previous labours have been immediately followed by hamorrhage.

Gateshead, Jan. 3, 1850.

\section{REPORT OF A CASE OF}

\section{DISEASED LARYNX, IN WHICH TRACHEOTONY} IVAS THREE TIMES PERFORAIED.

Br EDWIN HUMBY, EsQ., M.R.C.S., London.

Diseased Larynx; Tracheotomy performed three times; removal, by means of the Bone-Nippers, of several Ossified Rings of the Trachea; Expulsion of Ossified Cartilage by Coughing. A utopsy.

A $\mathrm{T}$ the request of the late Mr. Aston Key, I sent to the Medico-Chirurgical Society the accompanying report, which was read in abstract at the last meeting of the last session. As $I$ think it may prove to le interesting to many readers of ThE LANCET, I have sent it for publication in that journal.

T. $\mathrm{H}-$, aged fifty-three years, had passe? the greater part of his life at sea, and in the West Indies. In April, 1815, having had syphilis two years previously, he underwent a long course of mercury, to relieve secondary pains in the head, accompanied by frontal nodes, and, when convalescent, he went to Clifton, for change of air, where his health very much improved. In July following he canght a severe cold, had pains in the limbs, sore throat, loss of voice, and great difficulty of breathing. Bleeding, blistering, and other active treatment were used, without benefit, and tracheotomy was ultimately had recourse to. He wore a silver tube for one month, when, the breathing throngh the larynx being partially restored, it was dispensed with. Three weeks afterwards he returned to London: on the night of his return the breathing became so embarrassed that it was found absolutely necessary to reintroduce the tube. Under the advice of Mr. Aston Key he went to Brighton; was put under a course of mercury, by inunction, and was recommended to live in a room supplied with a constant jet of steam, in order to moisten the air previous to its entering the tube. His health very much improved under the mercury, but the passage through the larynx, above the artificial opening, remained impeded. Iodine was also tried, with out any more favourable result.

In July, 1846, having worn the tube ten months, and finding the difficulty of breathing increasing, he returned to London, for the purpose of obtaining further advice. He consulted $\mathbf{M r}$. Aston Key, Mr. Travers, Mrr. Teate, Mr. Lawrence, Mr. Liston, and Sir Philip Crampton, (who was then in London,) when it was concluded that the additional obstruction was caused by grauulations, which had sprung up about the inner opening of the tube, gradually pushing it out, and occasioning the increased difficulty of breathing. It was determined to enlarge the opening in the trachen downwards; and introduce a larger and longer tube.

On the 21st of July, Mr. Aston Key enlarged the wound, but found the rings of the trachea so ossified that he could not cut through them. In the evening, the breathing became more distressing; $\mathrm{Mr}$. Liston was sent for, who also tried to cut through the trachea, but was unable to do so. In the middle of the night, the symptoms getting more alarming, $\mathrm{Mr}$. Liston asked Sir Philip Crampton to visit the patient, with whose concurrence he cut out a large piece of the ossified rings of the trachea, with the bone-forceps, and introduced a larger tube. This gave great relief to the breathing; the next day the patient was enabled to walk about the room, and, soon afterwards, to go out in a carriage. He went on pretty com- fortably for three months, with the excoption of a slight attack of bronchitis.

In October, in consequence of the medical gentleman who constantly attended him having gone to Ireland, I was asked by Mr. Aston Key to take charge of the case. I now, for the first time, saw the patient; the previous history is almost ver. batim as he wrote it out himself.

He was, at this period, wearing a double tube, so that be could, before a looking-glass, (which he always found necessary to carry with him, remove the inner tube without disturbing the outer one, wash it, and replace it as often as required. He was suffering from constant congh, with mucopurulent fotid expectoration; of this, M[r. Liston once remarked, that it reminded him of the smell produced by the presence of a foreign body in the air tubes. He had hurried breathing; quick, weak pulse; great anxiety and irritability; restless nights, his sleep never exceeding one hour at a time. On removing the tube, and applying the hand over the opening in the neck, so as to close it, not the least breath appeared to pass through the larynx above the wound; a clear glass ap. plied to the mouth and nostrils was not dulled. The laryngeal voice was entirely gone, a slight noise alone was produced, by the motion of the lips, on attempting to speak. Over the left frontal protuberance was a portion of necrosed bone, the result of a node which had suppurated some months previously.

Dr. Williams was now called in consultation. On examination of the chest, the physical sigus were dulness and deficient breath sound, with tubular expiration in the left supra and infra spinous scapular regions, with weak vesicular breathing at the lower part of this side; muco-crepitation without dulness in the lower part of the right side.

The treatment consisted of blisters to the chest, demulcents and expectorants, anodynes at night, and nutritious diet. $\mathrm{He}$ lived in a suite of rooms on one floor, supplied with jets of steam in different parts to moisten the air, and assist in loosening the expectoration.

The cough getting daily more troublesome, the secretion more copious and purulent, and on several occasions the patient being very nearly suffocated from the tube being gradually more pushed out in consequence of the contraction of the external wound, it was determined to enlarge the opening downwards, and introduce a still larger tube.

On the 14 th of November, Mr. Liston cut out another piece of the ossified trachea with the bone forcens. After the operation, the patient was almost suffocated from the blood getting into the trachea, and during the struggle to recover his breath, he coughed up a large piece of necrosed ossified cartilage. On comparing it with a dried larynx, it resembled the posterior broad part of the cricoid cartilage.

Having got rid of this unexpected foreign body from the bronchus, we were in hopes that the cough and symptoms would be relieved, but too much disease had been already set up in the lungs, the breathing became shorter and more frequent, the pulse more rapid and small, the expectoration more purulent and fotid, and he gradually sank, and died six days after the operation.

Post-mortem examination.-An incision was made from the chin to the umbilicus, the sternum raised, the larynx and cavity of the chest exposed. The right pleural cavity contained about three pints of turbid serum, mixed with flakes of soft lymph. There was no disease of the right lung except moderate congestion.

The upper lobe of the left lung was adherent to the pleura, consolidated, of a pale reddish buff colour, exuding when squeezed a thick dirty red matter, a mixture of pus and blood, the texture readily breaking down, not crepitant, and sinking in water. In the left principal bronchus was found a small piece of necrosed ossified cartilage: the submucous texture of this bronchus was thick and yellow from infiltration of thick purulent lymph.

The passage through the larynx upwards, from the opening in the trachea, would just admit a fine probe, the posterior broad part of the cricoid cartilage was absent. The rings of the trachea were ossified nearly to its bifurcation, and there was a great contraction of its calibre around the opening which admitted the tube, from granulations projecting from the mucous membrane.

The preparation of the larynx was dissected by the late Mr. Potter, and placed in the museum of University College.

Remarks.-There is no reason to doubt that the origin of the disease was the chancre contracted rather more than two years previous to the first occurrence of symptoms in the larynx. The principal points of interest in the case, are, the 
great difficulty in making the opening into the trachea; (Mr. Liston told me it was the only occasion in which he had used the bone forceps for that purpose; also, the presence of a foreign body in the bronchus. How long the fragment of necrosed ossified cartilage had been there it is impossible to say, for I could trace no symptom to guide me; and although the peculiar smell of the expectoration has elicited from Mr. Liston the remark of its similarity to that caused by the presence of a foreign body in the air-tubes, and the dulness on percussion, and signs of pneumonia at the top of the left lung, proved that serious mischief was going on, yet we had no reason to suspect the presence of a foreign body, much less that a portion of necrosed ossified cartilage from the larynx had passed down into the bronchial tube. The presence of the small piece in the left bronchus at the post-mortem examination leaves little doubt that the part coughed up after the last operation had been there also.

In the treatment of the case, the greatest relief was found from keeping a constant supply of steam in different parts of the room, which answered the double purpose of moistening the dry warm air previous to entering the tube, and softening the thick muco-purulent expectoration that collected in and about it.

About a week before he died, he tried a tube contrived by Mr. Liston, with a half-valve at the orifice, so arranged as to admit a large quantity of air on inspiration, but only half that quantity on expiration: it was hoped that the air, being compelled to pass through the smaller orifice, would have expelled the sputa with less effort to the patient, but the disease in the lungs had too far advanced for this tube to be of any service. Mr. Liston had tried this plan with another patient he was attending at the same time, with great advantage and comfort.

Wlndsor-terrace, Maida-hill.

\section{ON CERTAIN IMPORTANT POINTS IN THE CHEMISTRY \& PATHOLOGY OF THE URINE.} By ARTHUR HILL HASSALL, M.B. Lond.

(Continued from p. 694, vol. ii. 18s9.)

A cid Phosplate of Soda.- This salt is of very frequent occurrence in the urine, in which it is oftentimes present in large quantities.

It usually crystallizes in combination with urea, yielding very characteristic crystalline forms, and on this account its recognition is of considerable pathological importance. I hope shortly to have some further observations to offer on this subject.

Basic Phosphate of Soda. - The rhombic or basic phosphate of soda is not unfrequently met with in a crystallized condition, and I have several drawings of modifications of its crystals.

Phosplhate of Solla and A mmonia.-This combination is also occasionally encountered in the urine, and its crystals may be readily recognised on the evaporation of a few drops of the urine containing it.

Phosplite of Magnesia.-This salt has hitherto, I believe, been noticed only in combination with ammonia; it occasionally occurs in a crystalline state by itself, and when suspected to exist, may be detected by the addition of ammonia, which converts it into the stellate bibasic salt.

Phosphate of Lime.-Doubts have been expressed as to whether this compound ever occurs in the urine in a crystalline condition. I have, however, frequently met with it in that state.

Sometimes it may be detected as a sediment in recent urine. More frequently it is met with in urine which has stood for some time, and which has undergone some degree of decomposition. The most abandoned formation of it which $I$ have yet noticed has been in saccharine and fermenting urine.

The crystals of this salt present themselves under several modifications, in plumes, bouquets, fans, and rosettes. Each of these modifications, when fully developed, is seen to be made up of a number of small and apparently wedge-shaped crystals. Occasionally it happens that the wedge-shaped crystals do not combine to form any definite and compound figure, but occur singly seattered through the urine.

One form of the phosphate of magnesia is represented in

Dr. Bird's " Urinary Deposits," woodcut 35, page 213.

I take this opportunity of expressing my obligations to Dr. Letheby, who, whenever $I$ am in doubt or difficulty, is kind enough to give me the benefit of his great knowledge and experience.

Notting Uill, Jan. 1850
REPORT OF A CASE OF

\section{LARGE PLICTLRED WOLXD OF THE RECTEM,} AND DISLOCATION OF THE COCCYX.

Br THOMAS O. DLICK, Esq, M.R.C.S., Islip, Oxon.

C. $\mathrm{H}-$, aged thirty-six, on sliding down a hay-rick, about wo o'clock P.M., came in contact with the handle of a hay fork sticking upright in the ground underneath, which penetrated the body to the depth of six inches, (as measured by the blood on the handle, which is three and a half inches in circum ference.) IIe was taken home, a distance of three miles, and at ten o'clock P.M. I saw him.

I found him in a state of collapse; pulse low, 62 , and feeble very sick and faint; had had some brandy-and-n ater several times, which he had ejected. I now administered aromatic spirit of ammonia with brandy at intervals, till he rallied. I then proceeded to an examination. There had been but very trifling hamorrhage. I found a punctured wound in the median line midway between the anus and sacrum, through which freces had passed. Upon inserting a finger into the wound, the dislocated coccr $x$ was felt loose in the perinæum and upon a second finger being introduced into the rectum per anum, they freely came in contact on the left side, at about the depth of three inches from each external opening. Convinced that extensive internal laceration existed, and considering that in his falling to the ground the apex of the handle would describe the segment of a circle, the extreme point of which I was unable to reach by the fingers, I introduced a full-sized bougie, and satisfactorily traced its progress by the lacerated side of the rectum, as far as the prostate gland. He had passed urine since the accident, showing the bladder and urethra intact. Reaction having taken place, I ordered a large poultice to the part, and to take solution of acetate of ammonia, with tiucture of henbaue, every four hours during the night.

June 19 th.-Ten A.M.: Has passed a restless night; complains of violent pain in perineo, and the lower part of the b. wels, with great difficulty of micturition; urine passes frequently, in small quantity; pulse 108, small and wiry; tongue dry at the edges, and red; great thirst; general irritability and restlessness; no faeces have passed. I bled him to twenty ounces; applied twenty-four leeches above the pubis; ordered hot flaunels to be assiduously applied to that region, and poultices to perinæum. To take chloride of mercury, potassiotartrate of antimony, with opium powder, every third hour, in full doses.-Nine P.M.: A decided improvement; pulse 98, more full, and softer; less tenderness in the pubic region; the inclination to pass urine not so frequent; the tiaces have passed involuntarily per anum and through the wound. A composing draught of Battley's solution at bedtime.

20th.--Improving. Continue medicine.

21st, 22nd, 23rd, and 24th.-Continues improving, (the chloride of mercury and potassin-tartrate of antimony have been gradually reduced to omission;) there is profuse discharge from the wound, and a circumscribed patch of inflammation in perineo, extending from the anus to the scrotum, with evident fluctuation, upon puncturing which, a considerable quantity of foetid pus, mixed with large flakes of sloughing cellular mombrane, escaped. To have disulphate of quinine and muriate of morphia, with port wine and beef-tea. This plan was continued to the 30 th, when another abscess appeared nearer the anus, which was then opened, and discharged the same kind of foetid pus as the former, and from which, on

July $4 \mathrm{th}, \mathrm{I}$ removed a large quantity of decayed cellular membrane. To continue quinine, and have porter and muttonchops, \&c. The faces still pass involuntarily, partly per anum, and partly through the posterior wound, which shows healthy granulation.

10th.-Gradually improving in every respect; has more power over the sphincter; the discharge more healthy.

17th.-Better. There appears a want of activity in the granulations of the anterior wound, with an unhealthy appearance of the intervening skin. I passed a director underneath it, and fully divided it. To apply resin ointment, and over that the poultice.

24 th. - The whole rapidly healing; healthy granulations and discharge; faces still pass through wound; more power in sphincter. Introduced a probe through wound, and finger per anum, when they met as before.

Aug. 8th.-Anterior portion of wound healed, except in one part; applied caustic; posterior much less, and except when fæces are liquid, none pass.

20 th. - Wound healed in anterior part; posterior as before; perfect control of sphincter. 